53 (2021) pp. 169-182

DOI: https://doi.org/10.33039/ami.2021.03.005

URL: https://ami.uni-eszterhazy.hu

\title{
Multi dimensional analysis of sensor communication processes ${ }^{*}$
}

\author{
Mohamed Amine Korteby, Zoltán Gál, Péter Polgár
}

\author{
University of Debrecen, Faculty of Informatics \\ korteby.amine@inf .unideb.hu \\ gal.zoltan@inf.unideb.hu \\ polgarp@mailbox.unideb.hu
}

Submitted: December 10, 2020

Accepted: March 8, 2021

Published online: May 18, 2021

\begin{abstract}
The Internet of Things requires communication mechanism to be optimal not only from the data transfer but from the energy consumption point of view, too. One of the most analyzed types of the sensor network is Low Energy Adaptive Clustering Hierarchy (LEACH) system depending on the population density, algorithm of cluster head election, heterogeneity of the energy and physical position of the nodes, velocity of the sink node, data aggregation rate and size of the data frame. Complexity of the system has been analyzed based on status data series of 360 simulation cases. New family of wireless sensor network (WSN) system is proposed with name CB-LEACH, having better characteristics than the classical LEACH system. The service ability of sensor network and dependency properties was done with analytic technique based on Singular Value Decomposition (SVD). Using this method there were identified most important modes serving as basis to regenerate responses of the studied sensor systems. It was found that the number of significant modes is just six. The novelty of the paper is a proof of concept that SVD is a useful multidimensional tool which can be used for describing the behavior of the newly proposed CB-LEACH family of sensor network mechanisms.
\end{abstract}

*This work was supported by the construction EFOP-3.6.3-VEKOP-16-2017-00002. The project was supported by the European Union, co-financed by the European Social Fund. This paper was supported also by the FIKP-20428-3/2018/ FEKUTSTRAT project of the University of Debrecen, Hungary and by the QoS-HPC-IoT Laboratory. 
Keywords: Wireless sensor networks, Low Energy Adaptive Clustering Hierarchy (LEACH), switching, cluster, classification analysis

AMS Subject Classification: 62H30, 68M10, 90B15, 62H25, 62H30

\section{Introduction}

Nodes in wireless sensor networks monitor an Area of Interest (AoI) and, depending on their function, data is transmitted directly or indirectly to a Base Station (BS) or Sink Node (SN) that is connected to a wired network for further processing. This forwarding process is accomplished through various routing mechanisms that are the focus of the related research. Because sensor devices have limited power resources, the efficiency of their energy consumption is crucial. At the same time, these sensors are heavily concentrated in physical areas that are difficult to access physically by human, so their power supply is virtually non-replaceable. Thus, the key to their operating time is the efficiency of their energy consumption.

Routing protocols play a key role in sending aggregated data, inducing careful handling of such tasks. A successful model of a WSN system is one that can strike a good compromise between the maximum amount of data collection and the minimum amount of energy consumption. In WSN routing mechanisms, clustering appears to be an important consideration as it provides efficient energy savings and data delivery at the network level. Hierarchical routing which includes clustering is proving to be a preferred method of arranging sensors [8, 16]. At the same time, the method increases scalability, reduces the amount of energy loss, and delay time, while providing good connectivity and load balancing with increased network life $[1,7]$.

LEACH is a hierarchical, cluster-based, energy-efficient routing mechanism. It extends the lifetime of the system with randomly selected Cluster Head $(\mathrm{CH})$ elements that forward frames from cluster members to the SN after aggregation. This intermediate transmission step significantly reduces the energy used by the nodes that operate the radio channel, since the transmission consumption of the data frame is based on the power function of the distance with exponent $2 b$. Here $b$ is the path loss exponent, its value depends on the path propagation properties and most often $b \geq 2$.

The further structure of the paper is as follows: in the second chapter we discuss the architecture and energy effects of the classical LEACH mechanism. In the third chapter we introduce our newly developed system Cost Balanced LEACH (CBLEACH) and we present the methods used for the analysis of multidimensional data sets. Chapter four examines the newly proposed WSN system based on a synthetic state data series generated using 360 different simulations. In the last chapter we summarize the results and formulate the directions for the possible continuation of the research work. 


\section{Architecture and functions of the LEACH mech- anism}

Routing protocols were divided into four schemes: Network Structure Scheme, Topology Based Scheme, Communication System Scheme, and Reliable Routing Schema. Besides, the network structure scheme can be broken down into two classes: Flat routing and Hierarchical routing, depending on the role of the sensor nodes. In Flat routing, the sensor nodes have similar roles and functionalities in the network. Used in small area networks, the principal issue of this type of routing is scalability. Flooding and Gossiping, Directed diffusion, Rumor, SPIN are the most popular Flat routing protocols.

Hierarchical routing delivers greater energy efficiency and reliability within its architecture, the entire network is partitioned into clusters and unique nodes called cluster heads $(\mathrm{CH})$ based on certain criteria. $\mathrm{CH}$ manages the gathering and aggregation of data received from its neighborhood, then forwards the collected data to the Base Station (BS) while providing other services to other nodes which consumes more energy. Hence is required cluster rotation method, a common approach often used to manage the energy harvesting inside the cluster.

LEACH is a Time Division Multiple Access (TDMA) based Media Access Control (MAC) routing protocol, self-adaptive and self-organized and most widely known hierarchical routing protocol. Due to its capability to increase energy efficiency it improves the lifespan of sensor nodes by using randomized rotations of local cluster head functions between the nodes $[11,12,14,18]$.

LEACH protocol consists of several rounds with two phases in each round: Set-up Phase and Steady Phase (see Figure 1).

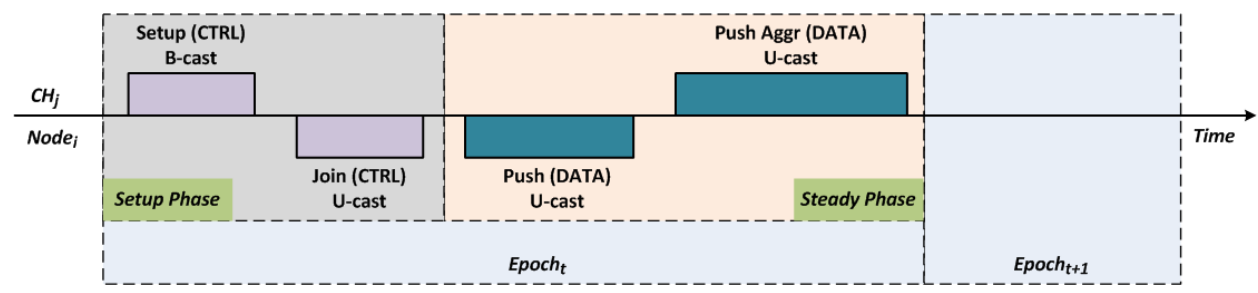

Figure 1. Mechanism for sending frames by epoch period.

In Set-up phase CH advertisement, cluster set-up, and TDMA scheduling are performed. During the $\mathrm{CH}$ advertisement, each node participates in $\mathrm{CH}$ election process based on the following equation:

$$
T(n)= \begin{cases}\frac{p}{1-p \cdot \bmod (r, 1 / p)}, & \text { if } n \in G, \\ 0, & \text { otherwise }\end{cases}
$$

where $T(n)$ is the threshold, $n$ is the node index taking values in continuous interval $[0,1]$ and set $\{1,2, \ldots, N\}$, respectively. Parameter $p$ denotes the $\mathrm{CH}$ election 
probability or the percentage of a node to become $\mathrm{CH}$ and is constant for a given simulation scenario. Function $\bmod (r, 1 / p)$ is the remainder after dividing the current index $r$ by the number $1 / p$ and $G$ is the set of sensor nodes that did not become $\mathrm{CH}$ in previous $1 / p$ rounds. Node $e_{n}$ acquires a cluster head function in the $r^{\text {th }}$ epoch period if it drew $q_{n}<T(n) . q_{n}$ is a random number $q_{n} \in 0,1$ drawn by Node $_{n}$ in the actual epoch. If $N_{\text {ode }}$ was $\mathrm{CH}$ in any of the last $1 / p$ epochs, then $T(n)=0$, so any $q_{n}>0=T(n)$, which means that it cannot regain a cluster head function in the actual epoch. With this rule and the random choice in the process, each node in the WSN is equal likely to become $\mathrm{CH}$ while achieving a homogeneous energy distribution among the CHs. The cluster head notifies all nodes in the system of its state with a control-type frame sent in a broadcast manner. Each of the nodes without $\mathrm{CH}$ function independently selects the most advantageous $\mathrm{CH}$ based on the field signal strength from the cluster head sources.

We refer round $r$ to be an epoch. A node with index $n$ becomes $\mathrm{CH}$ for the current round if the generated random value $q_{n}$ of a sensor node is strictly less than the threshold $T(n)$. For a given round we have a fixed threshold and it is compared to the sensor node random values. If it is equal to 0 , it means that $n$ does not belong to $G$ but belongs to the complementary set of nodes that were already elected as $\mathrm{CH}$ or died. Once a node is elected $\mathrm{CH}$ it cannot participate in the next $1 / p$ round of $\mathrm{CH}$ election: i.e. if $p \in\{0.05,0.10\}$ then the elected $\mathrm{CH}$ cannot be reelected in the next $1 / p \in\{20,10\}$ rounds respectively. This criterion is useful for the energy load balance inside the network since every node gets a better chance to become $\mathrm{CH}$.

Based on this rule any of the nodes can become $\mathrm{CH}$ with similar probability, providing uniformity on AoI space the extra energy consumption of the $\mathrm{CH}$ function. The CHs announce the other nodes with radio channel broadcast about its new $\mathrm{CH}$ function. The ordinary nodes receive these signals and based on the intensity of the signal decide which cluster to become a member with. The signal intensity in practice depends on different environmental parameters but for the classical version of the LEACH, just the distance between the node and the $\mathrm{CH}$ is considered. The ordinary nodes send their responses to the most advantageous $\mathrm{CH}$, becoming in this way members of that cluster. The $\mathrm{CH}$ schedules the communication inside of the cluster for the members during the actual epoch time.

In the epoch time second phase is named Seady Phase. During this period sensor end nodes send their data to the selected $\mathrm{CH}$. The $\mathrm{CH}$ aggreagates own frames with the transit traffic and send the aggregated data to the Sink Node. Thus, in the case of a WSN consisting of $\mathrm{N}$ sensor nodes, these two phases occur alternately in successive periods.

During the LEACH simulation, the strength of electromagnetic field is assumed to be exclusively distance-dependent, so the radio energy attenuation $A(d)$ per bit transmission is as follows:

$$
A(d)=E_{R x}(d) / E_{T x}(d),
$$




$$
A(d)= \begin{cases}1, & \text { if } d \in[0, \delta), \\ (\delta / d)^{2}, & \text { if } d \in\left[\delta, d_{0}\right), \\ \left(\delta / d_{0}\right)^{2} \cdot\left(d_{0} / d\right)^{2 b}, & \text { if } d \in\left[d_{0}, \infty\right),\end{cases}
$$

where $d$ is the distance between the sender and the receiver, $E_{R x}$ and $E_{T x}$ are the received and transmitted power, $\delta$ is the geometric parameter of the radio antenna (on the scale of centimeters), $d_{0}$ is the propagation distance threshold, $b$ is the path loss exponent, where $b \geq 2$. The node with its data selects the most advantageous $\mathrm{CH}$, i.e. the nearest $\mathrm{CH}$. In the absence of data, the node goes to sleep mode for an epoch period to save energy $[6,10]$. The $\mathrm{CH}$ provides a notification to the members of the cluster with a TDMA or Code Division Multiple Access (CDMA) control type frame, thus guaranteeing a collision-free frame transmission to the cluster head during the current epoch.

LEACH is the archetype for distributed routing protocols and one of the most efficient WSN mechanisms for power management. At the same time, we must not ignore some of its shortcomings [8]. The entry of any node into the $\mathrm{CH}$ function is independent of the residual energy of the given node, resulting in an uneven distribution in the physical location of the clusters in the covered area $[1,2,14$, 17]. This is exacerbated by the fact that the $\mathrm{CH}$ node may be even further away from the SN than members of its cluster, delivering data with poor efficiency [4, $5,15]$. Thus, some $\mathrm{CH}$ nodes drain their energy source sooner than other nodes, forming energy-free holes in physical space [3, 13]. Furthermore, the robustness of the network is reduced if the low-power node is given the $\mathrm{CH}$ function, because the frames of the cluster members arriving at the $\mathrm{CH}$ may be cancelled during the transmission to the SN due to the lack of power [9].

\section{Cost Balanced LEACH mechanism: CB-LEACH}

We propose a new family of LEACH mechanism named CB-LEACH having better performance than classical LEACH. With the change we proposed, we endowed the basic LEACH mechanism with additional skills and intelligence. This is a Cost Balanced (CB) version of $\mathrm{LEACH}$, which decides the route for transmitting frames based on complex metric. In the case of CB-LEACH, we allow the SN to move along a given path, as well as more efficient selection of the optimal $\mathrm{CH}$ for the end nodes. Because nodes in the current epoch time may be closer to the moving SN than the selected $\mathrm{CH}$, they should send their frames directly to the SN instead of indirectly through the selected $\mathrm{CH}$. To this end, we also include the SN in the set of selectable $\mathrm{CH}$ nodes. The energy of SN is considered not decreasing over time and is the largest in the WSN system. We consider not only the distance of the possible $\mathrm{CHs}$ from the end nodes but also their energy level, as well. To do this, a given sensor node decides own $\mathrm{CH}$ to connect to, based on the following metric:

$$
\operatorname{COST}(i, j, \alpha)=\alpha \cdot \frac{E_{0}}{E_{C H_{j}}}+(1-\alpha) \cdot\left(\frac{D(i, j)}{d_{0}}\right)^{2 b},
$$


where $\operatorname{COST}(i, j, \alpha)$ is the metric of $N_{o d e}$ and $C H_{j}$ calculated with a balance factor $\alpha \in[0,1], D(i, j)$ is the distance between $N_{o d e}$ and $C H_{j}, d_{0}$ is the propagation distance threshold and $b$ is the path loss exponent, $E_{0}$ is the initial energy for any normal type node (NN) and $E_{C H_{j}}$ is the actual energy level for a given $C H_{j}$. Parameter $\alpha$ named balance factor is our newly introduced element of the model to connect energy and distance properties of the nodes. Since the denominator of the first term of equation (3.1) can be zero after a long time, the properties of the system are analysed up to the last operating node, so that each term of the formula remains a positive and finite quantity.

In current epoch time $N_{0} e_{i}$ chooses the $\mathrm{CH}_{j}$ for which the value of the $\operatorname{COST}(i, j, \alpha)$ metric is the smallest. It can be observed that if $\alpha=0$, we get back the mobility-enhanced $\mathrm{CH}$ version of the LEACH mechanism, which is further identified as ENH-LEACH (Enhanced). If we do not change the position of the $\mathrm{SN}$ in time, we get back the Basic LECH mechanism, which is hereinafter referred to as BAS-LEACH.

However, if $\alpha=1$, only the energy of the $\mathrm{CH}$ counts, which generates a competitive situation between the potential $\mathrm{CHs}$ and each Node $_{i}$ will choose the same single $\mathrm{CH}$, i.e. the $\mathrm{SN}$ with the maximum energy. In this case, each node sends its data directly to the SN, independent of the other potential CHs. This routing mechanism will be referred to as DS (Direct Sequence) hereinafter.

Our simulation measurements demonstrated that ENH-LEACH and DS are significantly different routing protocols. The two extreme values of the balancing factor $\alpha$ have advantages and disadvantages from different aspects. When $\alpha$ is in range $(0,1)$ it has harmonization effect on the decisions of LEACH routing mechanism. Based on these, the proposed CB-LEACH is compatible with the family of protocols as follows:

$$
\text { CB-LEACH } \sim \begin{cases}\text { ENH-LEACH, } & \text { if } \alpha=0, \\ \text { Modified LEACH Familly, } & \text { if } \alpha \in(0,1), \\ \text { DS, } & \text { if } \alpha=1 .\end{cases}
$$

The DS sends the frame from each node directly to the SN. This means long distances, which consumes a significant amount of energy. However, due to the random transmission mechanism, the planar distribution of the residual energy of the nodes is expected to be strongly dependent on the distance between end node and SN. ENH-LEACH divides the physical field of the WSN into two parts, since treating the SN as a cluster head provides the nodes in the vicinity of the SN with a radius $d_{0}$ a favorable power consumption for frame transmission time. The obvious question is what properties the modified LEACH family inherits from the two boundary cases as a function of the $\alpha$ parameter. Further questions can put about the dependence of the CB-LEACH system on other parameters.

The CB-LEACH mechanism depends on a significant number of parameters, inducing execution of 360 simulation cases. In each case, the communication activity of a given WSN was completed during epoch numbers in the scale of $5 \cdot 10^{5}$. Vectorisation of the analyzed system responses is based on normalization of elemental 
answer vectors and concatenation of them in common vector named probe. There is a legitimate need to identify the parameters that most significantly influence the behavior of the present network. For this, we applied Singular Value Decomposition (SVD) analytic method [2], to efficiently evaluate behavior dependence on the tuplet of independent parameters. Having relatively high number of simulation probes, SVD gives possibility to determine the number of most significant modes of the CB-LEACH system behavior.

\section{CB-LEACH system analysis and characteristics}

We analyzed and evaluated the synthetic state data obtained from $n^{\prime}=360$ probes of Direct Sequence (DS), Basic LEACH (BAS-LEACH), and Enhanced LEACH (ENH-LEACH) simulations according to different parameters, using the methods described in this chapter. These mechanisms can be considered as special cases of CB-LEACH family proposed by us.

Figure 2 shows Area of Interest after ending the simulation of one probe example. Area of Interest is the biggest doted black circle. Red doted arc represents $d_{0}$ (see formula (2.2)), radio channel distance threshold. There are $N$ sensor nodes represented by black circles. Normal nodes (NN) and Advanced nodes (AN) are distinguished by the radius of black circles. Smallest circles belong to NNs and ANs have greater radius. For each simulation probe a number of $N$ sensor nodes are spread in uniformly distributed coordinates inside of AoI. Gray scale of the node marker is proportional with the age of node. Darker nodes die first, brighter nodes die later.

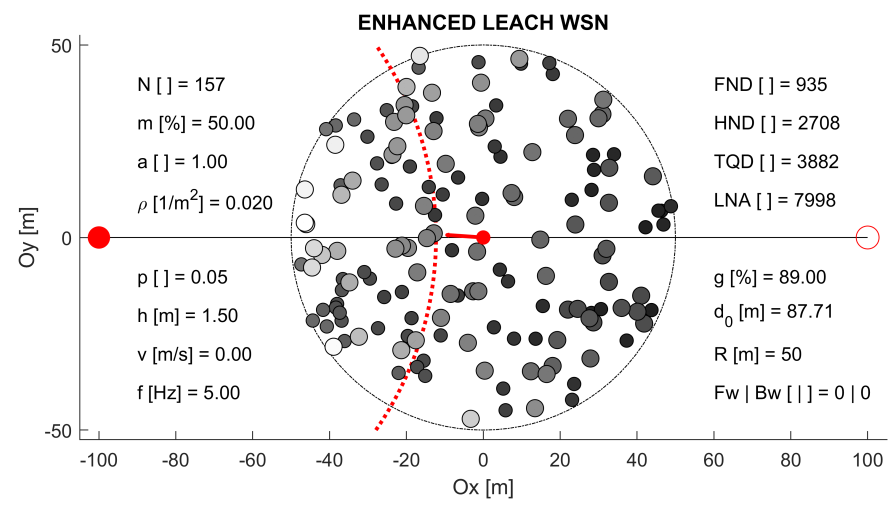

Figure 2. Example of one simulation probe after the execution of simulation. Parameter names conform to Table 1.

Depending on the constant velocity value, the sink node may move on trajectory in horizontal segment between filled red (left) and non filled (right) circles during the actual simulation. Number of forward and backward courses depend on the velocity and running time and are counted in variables $\mathrm{Fw}$ and $\mathrm{Bw}$, respectively. 
Being the sensor nodes uniformly distributed in space, the weighted energy point (WEP) in space of the WSN is situated in the center of the AoI. This WEP moves during the simulation if the remaining energy of the nodes is not uniformly decreasing in space. In this example red segment in the center zone of AoI represent movement of WEP during the simulation. FND, HND, TQD and LNA are epoch identifiers for first node die, half node die, third quarter node die and last node alive, respectively. Population density in the AoI is constant, $\rho=0.2 m^{-2}$ during all simulations, meaning one node per $5 \mathrm{~m}^{2}$.

The unchanged characteristics of the WSN system and the values of the six parameters of the simulation environment are shown in Table 1. In the table specific parameters have more than one value, others just one. Parameters with variable values are marked with star $\left(^{*}\right)$ character at the beginning of the line. Hereby we have $n^{\prime}=5 \cdot 3 \cdot 3 \cdot 2 \cdot 2 \cdot 2=360$ combinations of the orthogonal parameters giving us $n^{\prime}$ simulation probes.

Table 1. Parameters of the simulated system. Parameters having variable values are marked with star $(*)$ character at the beginning of the row. Other parameters have fixed value during the simulation probes.

\begin{tabular}{|l|l|}
\hline \multicolumn{1}{|c|}{ Parameters } & \multicolumn{1}{c|}{ Value $(\mathrm{s})$} \\
\hline Physical area size $(x \mathrm{xm} \times \mathrm{ym})$ & $100 \mathrm{~m} \times 100 \mathrm{~m}$ \\
\hline Radius of the field & $R=x m / 2$ \\
\hline Initial and farthest position of the Sink Node & $(-x m, 0)(\mathrm{xm}, 0)$ \\
\hline Number of nodes of the WSN & $N=157$ \\
\hline * Balance Factor & $\alpha=0,0.25,0.50,0.75,1.00$ \\
\hline * Ratio AN node number / total nodes, N & $m=0.3,0.5,0.7$ \\
\hline * Velocity of the Sink Node & $v=0,5,10 \mathrm{~m} / \mathrm{s}$ \\
\hline * Radio frames length & $F s=1000,4000 \mathrm{bits}$ \\
\hline * Aggregation level of the radio frames & $g=0.10,0.89$ \\
\hline * Ratio of the CH nodes & $p=5,10 \%$ \\
\hline Energy factor of the AN & $a=1$ \\
\hline Initial energy unit & $E_{0}=2.5 \mathrm{~J}$ \\
\hline Energy consumption of the electronics & $E_{e l e c}=50 \mathrm{~nJ} / \mathrm{bit}$ \\
\hline Energy multipath factor vs. of antenna height & $E_{m p}=1.3 \mathrm{pJ}$ \\
\hline Energy consumption of the antenna amplifier & $E_{a m p}=0.1 \mathrm{~nJ} / \mathrm{bit}$ \\
\hline Energy consumption of the frame aggregation & $E D A=5 \mathrm{~nJ} / \mathrm{bit}$ \\
\hline Radio antenna height & $h=1.5 \mathrm{~m}$ \\
\hline Radio channel distance threshold & $d_{0}=87.7 \mathrm{~m}$ \\
\hline Energy Free Space Factor & $E_{f s}=10 \mathrm{pJ} / \mathrm{bit} / \mathrm{m}^{2}$ \\
\hline Path loss exponent & $b=4$ \\
\hline
\end{tabular}

The CB-LEACH balancing factor $\alpha$ influences the cluster head selection strategy and the type of WSN routing based on equations (3.1) and (3.2). The cluster 
head average probability, $p$ plays a role in relation (2.1). Typically, the operation of the system is usually tested with relatively small values. To show the effect of heterogeneous initial energy levels, the set of $N$ sensor nodes are classified into two energy groups: NN (Normal Node) and AN (Advanced Node). AN initially has $a+1>1$ times greater energy than NN. The Ratio of the AN nodes number to the total nodes $N$ is $m \in(0,1)$. The initial energies of these two groups, and the entire WSN system, are as follows:

$$
\begin{aligned}
& E_{N}=(1-m) \cdot N \cdot E_{0}, \\
& E_{A}=m \cdot(a+1) \cdot N \cdot E_{0}, \\
& E_{T}=E_{N}+E_{A}=(a \cdot m+1) \cdot N \cdot E_{0} .
\end{aligned}
$$

Examples of status responses time series of WSN system for Basic LEACH can be seen on Figure 3. On the left hand side figure NN and AN node types have the spatial average energy level versus time represented with blue and green curves, respectively. Average energy curve for the whole WSN is shown by red curve. On the right hand side figure same colors are used to represent alive nodes versus the time.
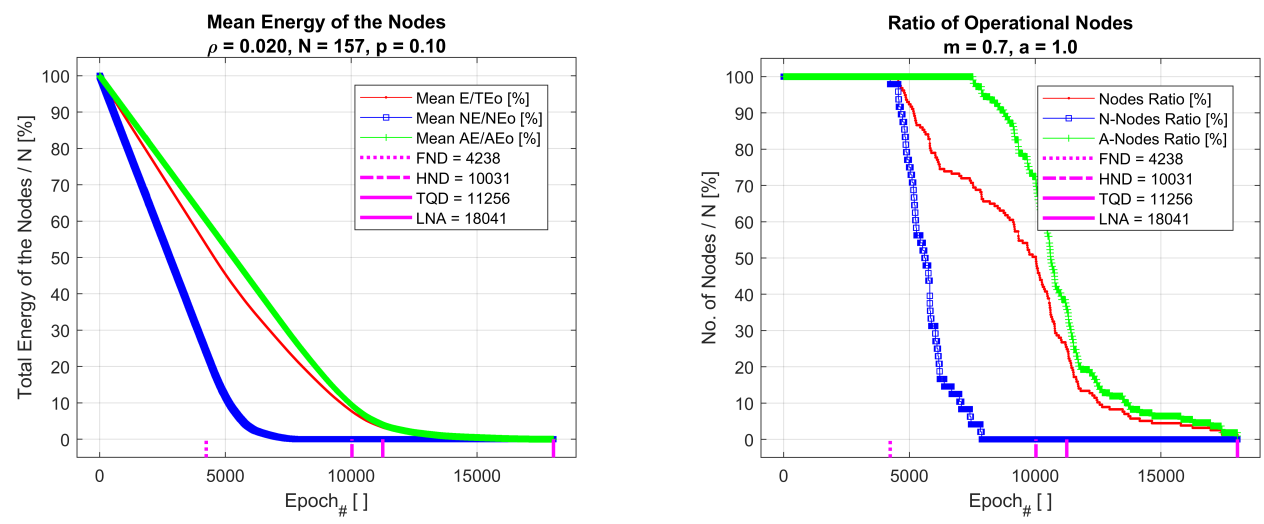

Figure 3. Examples of two elemental time series responses of Basic LEACH probe $_{i}$ : Remaining average energy level in space, $E_{i}^{B}$ (left) and Relative number of operational nodes, $N_{i}^{B}$ (right). Initial parameters are: $\alpha=0 ; m=0.7 ; v=0 \mathrm{~m} / \mathrm{s} ; F s=4000 \mathrm{bit} ; g=0.1$;

$$
p=0.1 \text {. }
$$

The response data series provided by the WSN system per routing mechanism are given by the following vector concatenations:

$$
\begin{aligned}
& y_{i}^{D}=\left(E_{i}^{D}, S_{i}^{D}, F_{i}^{D}, N_{i}^{D}\right) \in M_{1,8 e_{0}}, \\
& y_{i}^{B}=\left(E_{i}^{B}, S_{i}^{B}, F_{i}^{B}, N_{i}^{B}, T_{i}^{B}, K_{i}^{B}, D_{i}^{B}, R_{i}^{B}\right) \in M_{1,8 e_{0}}, \\
& y_{i}^{E}=\left(E_{i}^{E}, S_{i}^{E}, F_{i}^{E}, N_{i}^{E}, T_{i}^{E}, K_{i}^{E}, D_{i}^{E}, R_{i}^{E}\right) \in M_{1,8 e_{0}},
\end{aligned}
$$


where $M_{1,8 e_{0}}$ is the class of matrices with one row and $8 e_{0}$ columns. Upper indexes D, B and E stands for Direct Sequence, Basic LEACH and Enhanced LEACH mechanisms, respectively. Elemental responses of the WSN for simulation $i=$ $1, \ldots, m^{\prime}$ are vectors with $e_{0}$ elements each: spatial average remaining energy level in $\%\left(E_{i}\right)$, Shannon entropy in space of energy levels $\left(S_{i}\right)$, relative number of frames sent to the sink node in $\%\left(F_{i}\right)$, relative number of operational nodes in $\%\left(N_{i}\right)$, relative number of transactions/epoch in \% $\left(T_{i}\right)$, relative number of clusters/epoch in $\%\left(K_{i}\right)$, average distance in space between $\mathrm{CH}$ and sink node in $\%$ of $d_{0}\left(D_{i}\right)$, average cluster radius in $\%$ of $d_{0}\left(R_{i}\right)$.

Since we executed simulation for each combination of independent parameter values, every probe gives us response as a set of data series of the WSN system for DS, BAS-LEACH and ENH-LEACH (see Figure 4). Because number of elements of the response time series belonging to different probe are not equal, it was used dilatation and compression of the time. The common length $e_{0}=27,953$ of one elemental response vector is the average size of the elemental time series length of the $m^{\prime}$ probes, determined from Basic and Enhanced LEACH cases. In this way response vectors in formulae (4.1), (4.2) and (4.3) are transformed and considered as status vectors versus progress in the range $[0 \%, 100 \%)$.

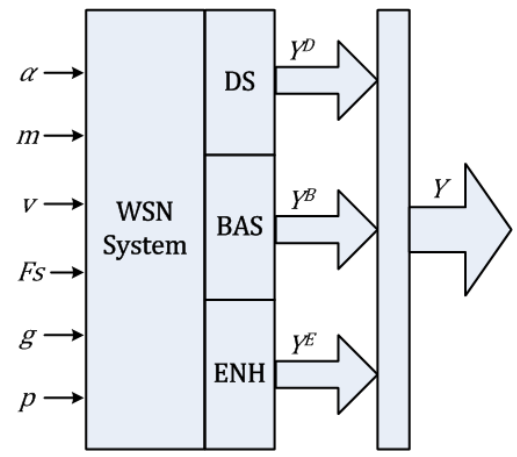

Figure 4. Structure of the WSN simulation system. For each parameter tuple the WSN system generates sensors in random positions of the AoI and executes simulation for DS, BAS-LEACH and ENH-LEACH routing mechanisms separately. Scaled and normalized response status data series are concatenated in probe vectors. Matrix $Y$ is the response matrix of WSN system having $n^{\prime}$ probes.

Each of the above three row-vectors $y_{i}^{D}, y_{i}^{B}, y_{i}^{D}$ contain $8 e_{0}$ elements. Because in case of DS mechanism just $E^{D}, S^{D}, F^{D}, N^{D}$ response data series have meaning, being half as many time series as BAS-LEACH or ENH-LEACH mechanisms have, each DS vector mentioned was scaled to double length, $2 e_{0}$. Each elemental status data series of probe $y_{i}, i=\overline{1, n^{\prime}}$ generated by simulation $i$ of the WSN system is concatenated to create the probe vector identified by the following formula:

$$
y_{i}=\left(y_{i}^{D}, y_{i}^{B}, y_{i}^{E}\right)^{T} \in M_{m^{\prime}, 1},
$$


where $T$ is the transpose operator. Collection of column vectors $y_{i}, i=\overline{1, n^{\prime}}$ represents status matrix of the WSN system given by the following formula:

$$
Y=\left(y_{1}, \ldots, y_{n^{\prime}}\right) \in M_{m^{\prime}, n^{\prime}},
$$

where $m^{\prime}=(2 \cdot 4+8+8) \cdot e_{0}=24 \cdot e_{0}=670,872$ and $n^{\prime}=360$.

Should mention that since the structure of the $y_{i}$ column vector representing the simulation probe $i$ is fixed, it can be considered as a vectorized image map. Each of the $n^{\prime}$ different probes is a composed object of twenty vectorized images. Each object belonging to the corresponding probe has the same structure but different content from the others. Hereby we have better representation of the system responses and we search a smaller number of representative probes, $k$ that are able to best characterize the WSN system.

We used Singular Value Decomposition to determine the number of most important modes able to form an orthogonal basis for all the $n^{\prime}$ probes. According to Figure 5 (left), the correlation coefficients between the responses $y_{i}$ and $y_{j}$ are in the interval $(0.5,0.95)$, mostly closer to the larger values, where $i<j, i, j \in\left\{1, \ldots, n^{\prime}\right\}$. It is numerically confirmed by Figure 5 (right), that the $k=6$ largest singular values represents $33.55 \%$ of the information given by $n^{\prime}=360$ singular values. The elbow part of the singular values scree plot proves that there are $k=6$ virtual modes serving as synthesization basis for all $n^{\prime}=360$ probes. It means that there are 6 different virtual parameter tensor values $(\alpha, m, v, F s, g, p)$, which represent 6 virtual probes that primarily characterize our CB-LEACH WSN system. This is considered the main novelty of our findings about the proposed CB-LEACH family of routing mechanisms.
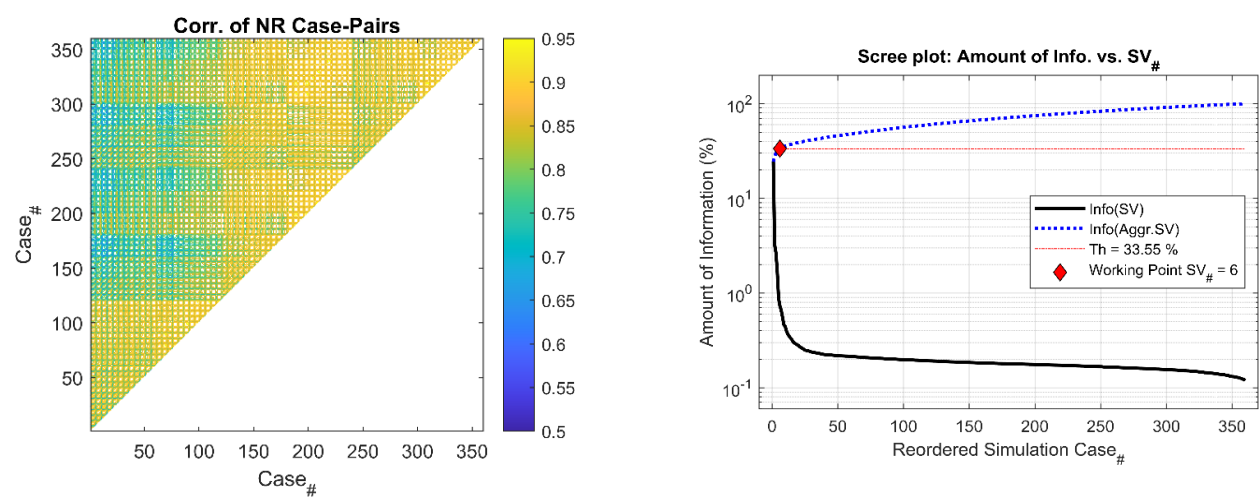

Figure 5. Correlation matrix of Y responses of WSN system (left) and singular values of the response matrix Y (right).

Decomposition of status matrix $Y$ is given by the following formula:

$$
Y=U * \Sigma * V^{T}
$$

where matrixes $U \in M_{m^{\prime}, m^{\prime}}, \Sigma \in M_{m^{\prime}, n^{\prime}}$ and $V \in M_{n^{\prime}, n^{\prime}}$ are unitary basis in progress, singular value matrix and unitary basis matrix in AoI space, respectively. 
The simplified response matrix $\hat{Y}$ is the approximation of the matrix $Y$ and contains in each column 20 elementary data series having the noise reduced significantly:

$$
\hat{Y}=U_{k} * \Sigma_{k} * V_{k}^{T}
$$

where matrixes $U_{k} \in M_{m^{\prime}, k}, \Sigma_{k} \in M_{k, k}$ and $V_{k} \in M_{n^{\prime}, k}$ are most significant $k$ columns of matrixes $U, \Sigma$ and $V$, respectively.

Figure 6 demonstrates similarity between the original $(Y)$ and filtered response system $(\hat{Y})$. Both parts of Figure 6 contain 7,200 data series versus progress. The response data series belonging to a given probe contains the values going from top to bottom along with the columns. The values of the normalized and scaled response data series are represented by color codes having values conform to the color bars. It can be observed that the two images are very similar in both aspect of layout and sharpness. The root mean square error (RMSE) of the two matrices is $R M S E=9.15 \%$.
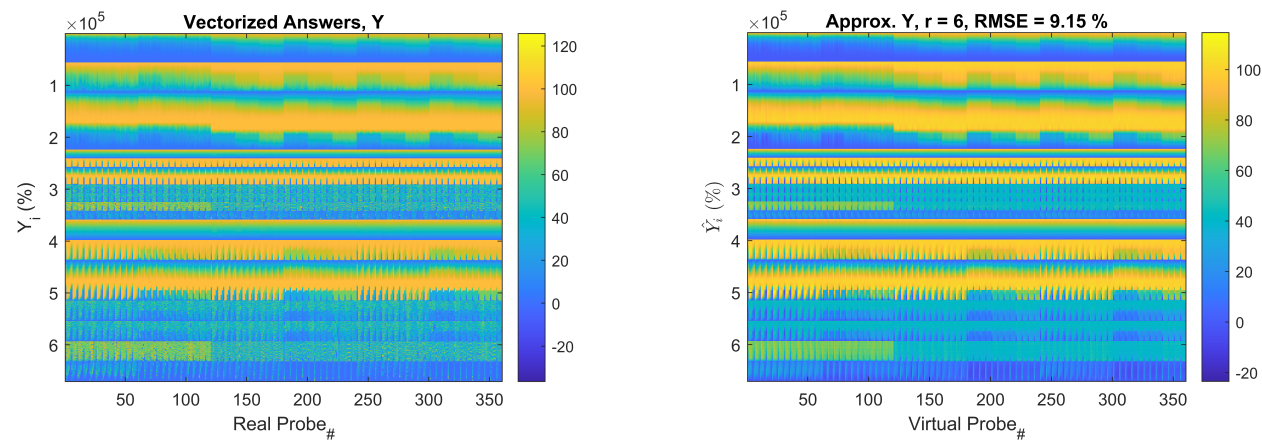

Figure 6. Data series responses of a WSN system, original $Y$ (left) and approximated $\hat{Y}$ (right). Progress direction is from top to bottom, normalized values are represented by color codes.

The facts found so far prove the feedback neural networks to be useful in analysis of complex systems as they reduce the learning process required for modelling. This aspect is not discussed in this paper because it is subject of our next research phase. Using the $k$-means clustering method, we classified the responses of the $n^{\prime}$ simulations cases (probes) into $k$ classes, i.e. $y_{i}, i=\overline{(1, n)}$ column vectors into clusters. The result of this computation is illustrated in Figure 7 . The number of probes belonging to groups $1, \ldots, k$ are $96,72,96,24,38$ and 34 , respectively (see Figure 7 left). Since the number of members is of the similar order of magnitude per class, each class is important. Figure 7 (right) represents centroid vectors of $k=6$ clusters conform to probes classification provided by the $k$-Mean algorithm. Centroid of a class is the mean vector in space for member vectors belonging to the same class. We should mention that significant difference exists between these centroid vectors. 

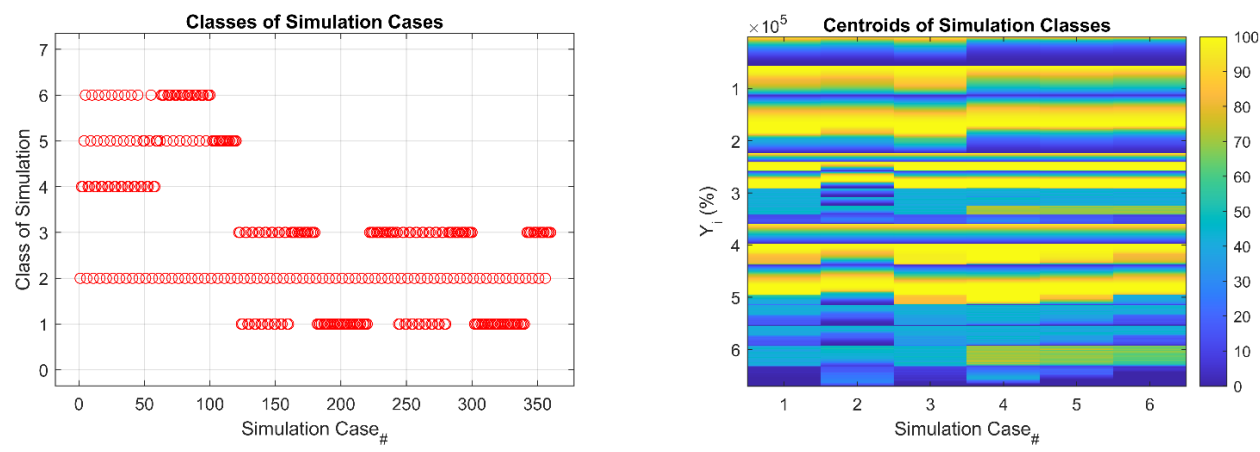

Figure 7. Classification of simulations by $k$-Mean algorithm into $k=6$ clusters (left) and centroids of the probe classes (right).

This aspect proves detectable diversion between the probe classes resulted for simulations based on $n^{\prime}$ tuple of independent parameters of the proposed CBLEACH family of WSN mechanisms.

\section{Summary and conclusions}

The method presented in the paper allows the behaviour analysis of the newly proposed CB-LEACH wireless sensor routing mechanism. CB-LEACH is an energyefficient extension of the classic LEACH, the operation of which can be influenced by six parameters. To determine the optimal parameter tensor, the analysis of the synthetic state data set was generated by 360 different simulation cases which required the use of dimension reduction analysis based on Singular Value Decomposition to determine the number of most significant modes. This number is $k=6$, which is not a coincidence due to the number of independent parameters $(\alpha, m, v, F s, g$ and $p)$, but generic property due to the special behaviour of the CBLEACH family. To classify the $n^{\prime}=360$ simulation cases into $k$ classes, we used $k$-Mean clusterization algorithm enrolling to the closest cluster with good accuracy each simulation probe based on independent tuples of parameters.

As a continuation of the research work, further analyzes are considered important on the noise reduction of response probes of elementary data series as a function of singular value number and reducing precessing capacity of the method. Sensitivity analysis will be executed to find approximated closed-from expression of the energy usage dependence on the multidimensional parameter set.

\section{References}

[1] O. M. Alia: Dynamic relocation of mobile base station in wireless sensor networks using a cluster-based harmony search algorithm, Information Sciences (2017), pp. 76-95, DOI: https://doi.org/10.1016/j.ins.2016.12.046. 
[2] S. Baljinder, V. Amit, K. Manit: A survey on various energy-efficient routing protocols in WSN, International Journal of Advanced Research, Ideas and Innovations in Technology 4 (2019), pp. 862-865.

[3] M. BelwAL: Energy Efficient LEACH and Improved LEACH: A Review, International Journal of Advanced Research in Computer Science 10 (2019), pp. 51-52, DOI: https://doi.org/10.26483/ijarcs.v10i3.6412.

[4] Y. Chi, H. Chang: An energy-aware grid-based routing scheme for wireless sensor networks, Telecommunication Systems 54 (2013), pp. 405-415, DOI: https://doi.org/10.1007/s11235-013-9742-x.

[5] Z. Gal, M. Konteby: Energy Sparing of the Leach Communication Mechanism in Heterogeneous WSN, in: 8th International Conference on Advanced Science and Information Technology, 2019, pp. 53-64.

[6] N. Ghosh, I. Banerjee: An energy-efficient path determination strategy for mobile data collectors in wireless sensor network, Computers \& Electrical Engineering 48 (2015), pp. 417435, DOI: https://doi.org/10.1016/j.compeleceng.2015.09.004.

[7] S. Harjit, S. VArun: Energy Efficient Clustering for Network Stability and Longevity for Heterogeneous Wireless Sensor Network, International Journal of Engineering Science and Computing 8 (2018), pp. 18867-18872.

[8] W. Heinzelman, A. Chandrakasan: An application-specific protocol architecture for wireless micro sensor networks, IEEE Transactions on Wireless Communications 1 (4 2002), pp. 660-670, DOI: https://doi.org/10.1109/TWC.2002.804190.

[9] N. Ismat, R. Qureshi, I. Mumtaz: Efficient Clustering for Mobile Wireless Sensor Networks, in: 17th IEEE International Multi Topic Conference, 2014, pp. 110-114, DOI: https://doi.org/10.1109/INMIC.2014.7097321.

[10] A. Khan, A. Abdullah, M. Razzaque, J. Bangash: Vgdra: A virtual gridbased dynamic routes adjustment scheme for mobile sink-based wireless sensor networks, IEEE Sensors Journal 15 (2015), pp. 526-534.

[11] J. Kim, J. In, K. Hur, J. Kim, D. Eom: An intelligent agent-based routing structure for mobile sinks in WSNs, IEEE Transactions on Consumer Electronics 4 (2010), pp. 2310-2316.

[12] W. Liang, J. Luo, X. Xu: Prolonging network lifetime via a controlled mobile sink in wireless sensor networks, in: IEEE global telecommunications conference GLOBECOM, 2010, pp. 1-6.

[13] N. Mittal, U. Singh, B. Sohi: A stable energy efficient clustering protocol for wireless sensor networks, Wireless Networks 23 (2017), pp. 1809-1821.

[14] H. Salarian, K. Chin, F. Naghdy: An energy-efficient mobile-sink path selection strategy for wireless sensor networks, IEEE Transactions on Vehicular Technology 63 (2014), pp. 2407-2419.

[15] M. Thakur: Mobile Sink Based NLEACH Protocol by using Ant Colony Optimization, International Journal of Science Research and Technology 2 (2016), pp. 1-10.

[16] J. WAng, Y. CAO, B. Li, H. Kim, S. LeE: Particle swarm optimization based clustering algorithm with mobile sink for WSNs, Future Generation Computer Systems 76 (2016), pp. 452-457, DOI: https://doi.org/10.1016/j.future.2016.08.004.

[17] M. A. Zahhad, S. Ahmed, N. Sabor, S. SAsaki: Mobile sink-based adaptive immune energy-efficient clustering protocol for improving the lifetime and stability period of wireless sensor networks, IEEE Sensors Journal 15 (2015), pp. 4576-4586.

[18] Z. Zhou, C. Du, L. Shu, G. Hancke, J. Niu, J. Ning: An Energy-Balanced Heuristic for Mobile Sink Scheduling in Hybrid WSNs, IEEE Transactions on Industrial Informatics 12 (2016), pp. 28-40. 